\title{
Emergent scale invariance of nonclassical plasmons in graphene nanoribbons
}

\author{
Wedel, Kåre Obel; Mortensen, N. Asger; Thygesen, Kristian Sommer; Wubs, Martijn
}

Published in:

Physical Review B

Link to article, DOI:

10.1103/PhysRevB.98.155412

Publication date:

2018

Document Version

Publisher's PDF, also known as Version of record

Link back to DTU Orbit

Citation (APA):

Wedel, K. O., Mortensen, N. A., Thygesen, K. S., \& Wubs, M. (2018). Emergent scale invariance of nonclassical plasmons in graphene nanoribbons. Physical Review B, 98(15), [155412].

https://doi.org/10.1103/PhysRevB.98.155412

\section{General rights}

Copyright and moral rights for the publications made accessible in the public portal are retained by the authors and/or other copyright owners and it is a condition of accessing publications that users recognise and abide by the legal requirements associated with these rights.

- Users may download and print one copy of any publication from the public portal for the purpose of private study or research.

- You may not further distribute the material or use it for any profit-making activity or commercial gain

- You may freely distribute the URL identifying the publication in the public portal

If you believe that this document breaches copyright please contact us providing details, and we will remove access to the work immediately and investigate your claim. 


\title{
Emergent scale invariance of nonclassical plasmons in graphene nanoribbons
}

\author{
Kåre Obel Wedel, ${ }^{1,2,3}$ N. Asger Mortensen, ${ }^{3,4,5}$ Kristian S. Thygesen, ${ }^{2,3}$ and Martijn Wubs ${ }^{1,3}$ \\ ${ }^{1}$ Department of Photonics Engineering, Technical University of Denmark, Ørsteds Plads, Bldg. 345A, DK-2800 Kongens Lyngby, Denmark \\ ${ }^{2}$ Department of Physics, Technical University of Denmark, Fysikvej, Bldg. 307, DK-2800 Kongens Lyngby, Denmark \\ ${ }^{3}$ Center for Nanostructured Graphene (CNG), Technical University of Denmark, Ørsteds Plads, Bldg. 345C, \\ DK-2800 Kongens Lyngby, Denmark \\ ${ }^{4}$ Center for Nano Optics, University of Southern Denmark, Campusvej 55, DK-5230 Odense M, Denmark \\ ${ }^{5}$ Danish Institute for Advanced Study, University of Southern Denmark, Campusvej 55, DK-5230 Odense M, Denmark
}

(Received 13 July 2018; published 12 October 2018)

\begin{abstract}
Using a nearest-neighbor tight-binding model we investigate quantum effects of plasmons on few-nanometer wide graphene nanoribbons, both for zigzag and armchair edge terminations. With insight from the Dirac description we find an emerging scale-invariant behavior that deviates from the classical model both for zigzag and armchair structures. The onset of the deviation can be related to the position of the lowest parabolic band in the band structure. Dirac theory is only valid in the parameter subspace where the scale invariance holds that relates narrow ribbons with high doping to wide ribbons with low doping. We also find that the edge states present in zigzag ribbons give rise to a blue shift of the plasmon, in contrast to earlier findings for graphene nanodisks and nanotriangles.
\end{abstract}

DOI: 10.1103/PhysRevB.98.155412

\section{INTRODUCTION}

Since its discovery [1], graphene has attracted much attention in the scientific community, initially mainly for its remarkable electronic properties as well as its unprecedented mechanical qualities [2]. However, the plasmonic capabilities of this conveniently tunable material have also received great interest in recent years [3-9] along with other twodimensional (2D) materials [10]. As ever smaller and more precise devices [11-15] are produced, it is important to obtain corresponding theoretical understanding of plasmons in graphene nanostructures. For instance nanodisks and nanotriangles have both been investigated both theoretically [16-19] and in experiments [20], and more complex structures have also been studied [21-23].

The electronic properties of graphene nanostructure can be described on various levels of sophistication. Classically, it is a finite-size conductivity sheet. The simplest atomistic description is a tight-binding (TB) model for the electrons. The Dirac-equation continuum model for finite graphene structures is of intermediate complexity and describes lowenergy electrons with linear dispersion being confined on finite graphene structures. Each of these three electronic models has its associated optical response, so that plasmonic excitations may also vary. While the tight-binding model is the most microscopic of them, it is important to know when the simpler Dirac or even the classical description suffices, and for which parameters the three models start to deviate from each other, and how important for optical properties are the different electronic edge terminations $[24,25]$.

In this paper we present quantum-mechanical calculations of graphene nanoribbons, with geometries as depicted in Fig. 1, in particular tight-binding calculations in the randomphase approximation (RPA). Important previous work on this topic includes theoretical contributions both for isolated ribbons and for arrays of them [26-33], as well as experimental studies [34-39] with ribbon widths down to $15 \mathrm{~nm}$ [40]. Furthermore, in a complementary analytical analysis we identify a scale invariance in the Dirac-equation model for graphene ribbons [41], a scale invariance that it shares with the classical model but not the tight-binding model. For the latter we identify the scale invariance as an emergent property. Thereby we obtain an illuminating overview for which parameters the Dirac-equation model can agree with the tight-binding models. Furthermore, we identify a scale invariant onset of quantum mechanical effects.

The paper is structured as follows. In Sec. II we briefly discuss the TB model and its numerical evaluation, and the corresponding optical response function in terms of the electronic states. In Sec. III we review the Dirac-equation model both for zigzag and armchair graphene ribbons, use the band structures to identify the onset of nonclassical effects, and we identify the dimensionless scaling behavior property. In Sec. V we compare our numerical TB calculations with our analytical predictions, and we conclude in Sec. VI. Detailed information can be found in two Appendixes.

\section{NUMERICAL METHODS}

\section{A. Tight-binding model}

We describe the graphene ribbon in a nearest-neighbor tight-binding model with the Hamiltonian

$$
H=\sum_{\langle i, j\rangle}-t\left(a_{i}^{\dagger} b_{j}+\text { H.c. }\right),
$$

where the sum is over pairs of neighboring sites. This model has proven useful for describing the band structure in a wide energy range around the Dirac point as the bands here are determined by interaction between the $p_{z}$ orbitals of the $s p^{2}$ 


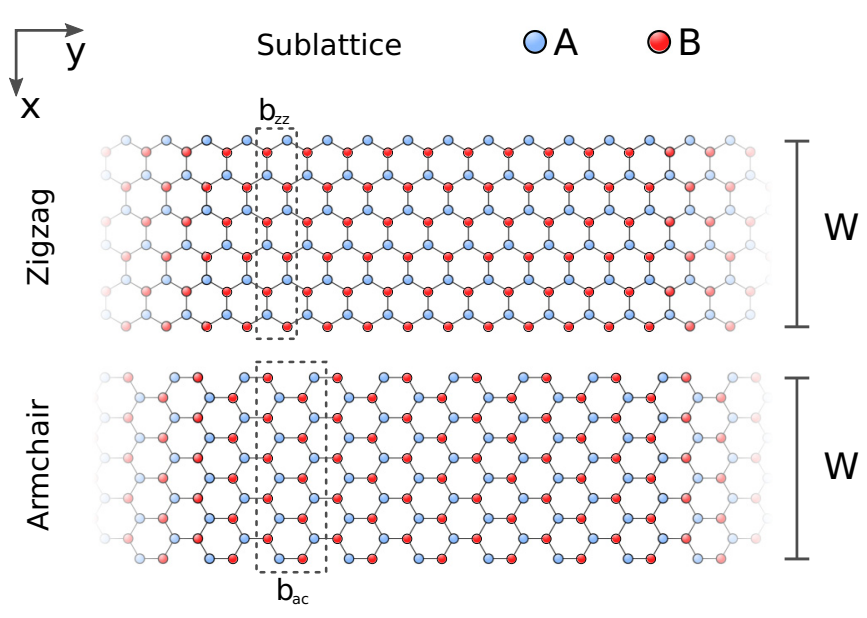

FIG. 1. The geometries of the zigzag and armchair graphene ribbons. The supercells are marked with the dashed rectangles.

hybridized carbon atoms. A hopping value of $t=2.8 \mathrm{eV}$ is used between all interacting atoms as it has generally been found to give good results [42].

We have used the smallest possible supercell, which includes one row of atoms for the zigzag (ZZ) ribbons and two rows for the armchair (AC) ribbons as illustrated in Fig. 1. The band structure and the states are found by direct diagonalization of the Hamiltonian with a $k$-point sampling of at least 5000 points in the Brillouin zone, which has been found to give converged results in the subsequent evaluation of the optical response.

In Fig. 2 we show the bands around the $K$ point for two $6 \mathrm{~nm}$-wide ribbons, one with $\mathrm{ZZ}$ and the other with $\mathrm{AC}$ edge terminations. The dots correspond to TB calculations, and the colors indicate the edginess (defined in Appendix A) of the

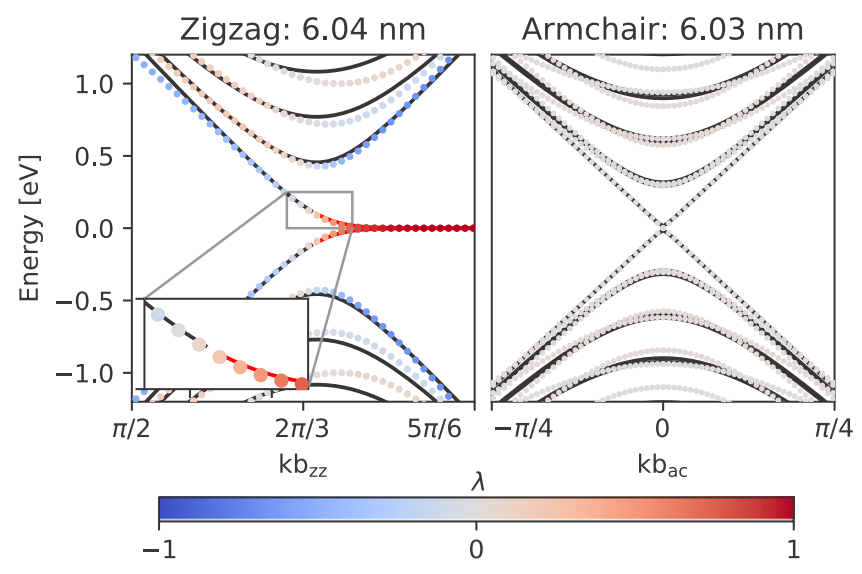

FIG. 2. Band structures of $6 \mathrm{~nm}$-wide zigzag and armchair ribbons. Full lines are the bands obtained with the Dirac theory, with red color indicating the edge states. The colored dots are the corresponding energies as calculated with the TB model. The color bar indicates how edgelike the states are, as defined in Eq. (A1), with the value $\lambda=1$ corresponding to a state localized on the edges and -1 to a state localized in the center of the ribbon. The armchair states are uniformly distributed across the ribbon and thus satisfy $|\lambda| \ll 1$ for all states. corresponding states, with bright red corresponding to an edge state. The figure also shows the continuous bands calculated within Dirac theory, as discussed in Sec. III.

\section{B. Response function and quantum plasmons}

The optical response of a quantum mechanical system is described in full by the dielectric function, which relates to the noninteracting electron density-density response operator $\chi^{0}$ in the RPA as $[43,44]$

$$
\epsilon_{i j}(\omega)=\delta_{i j}-\sum_{m} V_{i m} \chi_{m j}^{0}(\omega)
$$

where $V_{i j}$ is the Coulomb interaction. Following the method of Ref. [26], $\chi^{0}$ is calculated from the TB eigenstates, in the case of only vertical excitations, i.e., neglecting intraband transitions, as

$$
\chi_{i j}^{0}(\omega)=\frac{2 e^{2}}{\hbar} \frac{b}{2 \pi} \int^{\mathrm{BZ}} d k \sum_{n m} f_{n m} \frac{a_{i n} a_{i m}^{*} a_{j n}^{*} a_{j m}}{\epsilon_{n m}+\hbar(\omega+i \eta)}
$$

Here the $i, j$ run over atomic sites, while $n, m$ label the eigenmodes at wave vector $k$. Thus, $a_{i n}$ is the weight of the $n$th wave function on the $i$ th site (implicitly at wave vector $k$ ). We have used the shorthand notation $\epsilon_{n m}=\epsilon_{n}-\epsilon_{m}$ for the energy difference and similarly for the Fermi filling factors $f$. Damping is included phenomenologically through the parameter $\eta$, which we set to $1.6 \mathrm{meV}$ throughout as in Ref. [26]. The parameter $b$ is the width of the supercell in the periodic direction, see Fig. 1.

From the density-density response function we calculate the dielectric matrix $\epsilon(\omega)=I-V \chi^{0}(\omega)$. This expression involves the Coulomb interaction $V$ in real space, which is a subtle matter to handle [26], both due to its long-range behavior and because of the divergence at zero distance, but it can be done (details in Appendix B). As shown in Ref. [45], $\epsilon(\omega)$ can be written in a spectral representation of its eigenvalues and left and right eigenvectors as

$$
\epsilon_{i j}(\omega)=\sum_{n} \epsilon_{n}(\omega) \phi_{n, i}(\omega) \rho_{n, j}^{*}(\omega)
$$

where the $i, j$ are again site indices in the tight-binding basis and the $\epsilon_{n}(\omega)$ the eigenvalues; the right eigenvector $\phi_{n}$ is the induced field, and the left eigenvector $\rho_{n}$ is the induced charge of the plasmon. The zeros of the real parts of $\epsilon_{n}(\omega)$ define the plasmonic modes. In this work, we concern ourself with the dipole mode, i.e., the plasmon with the lowest-lying energy. There will be plasmons at higher energies as well, corresponding to higher-order modes in the ribbon. The zeros of $\Re\left(\epsilon_{n}\right)$ agree well with peaks in the energy-loss function $-\operatorname{Im} \epsilon^{-1}(\omega)$ as measured in electron energy-loss spectroscopy experiments, provided the frequency dispersion of the imaginary part of $\epsilon_{n}(\omega)$ is small. The above method to calculate quantum plasmons based on a tight-binding formalism will be applied to graphene ribbons in Sec. II B. 


\section{ANALYTICAL MODEL}

\section{A. Dirac theory for graphene ribbons}

Dirac theory is an approximate theory obtained by linearizing the TB model in the $K\left(K^{\prime}\right)$ valleys where infinite graphene exhibits its Dirac cones. This allows one to get analytical insight into the band structure also of finite graphene structures. For graphene ribbons, this was first done in the seminal paper by Brey and Fertig [41] and the method is also outlined in Castro Neto et al. [42]. Here we first briefly review the Dirac theory, before presenting our new analytical insights and their comparison with full TB calculations.

In its essence, in the low-momentum limit the tight-binding Hamiltonian is approximated as

$$
\begin{aligned}
H & =\hbar v_{\mathrm{F}}\left(\tau_{0} \otimes \sigma_{x} k_{x}+\tau_{z} \otimes \sigma_{y} k_{y}\right) \\
& =\hbar v_{\mathrm{F}}\left(\begin{array}{cccc}
0 & k_{x}-i k_{y} & 0 & 0 \\
k_{x}+i k_{y} & 0 & 0 & 0 \\
0 & 0 & 0 & -k_{x}-i k_{y} \\
0 & 0 & -k_{x}+i k_{y} & 0
\end{array}\right),
\end{aligned}
$$

where $\tau_{i}$ and $\sigma_{i}$ are the Pauli spin matrices belonging to the valley space and sublattice space, respectively. For the eigenstates of the system we adopt the notation of Ref. [41]: $\left[\phi^{A}, \phi^{B},-\phi^{A^{\prime}},-\phi^{B^{\prime}}\right]^{T}$. The Hamiltonian in Eq. (5) is block diagonal, so we focus only on the upper left corner corresponding to the $K$ valley. By applying $H$ twice to a state $\left[\phi^{A}, \phi^{B}\right]^{T}$ we find the relations $\left(k_{x}^{2}+k_{y}^{2}\right) \phi^{A / B}=\tilde{\epsilon}_{k}^{2} \phi^{A / B}$ with $\tilde{\epsilon}=\epsilon / \hbar v_{\mathrm{F}}$.

By replacing $k_{x}$ with $-i \partial_{x}$, a differential equation is obtained with the general solutions

$$
\phi^{X}(x)=A e^{\beta x}+B e^{-\beta x},
$$

with $\beta=\sqrt{k_{y}^{2}-\tilde{\epsilon}^{2}}$, and consequently $\epsilon=s \hbar v_{\mathrm{F}} \sqrt{k_{y}^{2}-\beta^{2}}$ where $s= \pm 1$. The eigenmodes of the Hamiltonian can be found analytically for both ribbon geometries of Fig. 1 by imposing proper corresponding boundary conditions for their wave functions. These boundary conditions are different for zigzag and for armchair edge terminations.

\section{Zigzag edge termination}

In a $\mathrm{ZZ}$ ribbon the atomic structure terminates on an $A$ lattice site on one edge and on a $B$ site on the opposite edge, see Fig. 1. The proper boundary conditions, $\phi^{A}(x=$ $0)=\phi^{B}(x=W)=0$, lead to the dispersion relation for the allowed states in a slightly different notation than in Ref. [41]:

$$
k_{y}=\frac{\beta}{\tanh (\beta W)} .
$$

For fixed $k_{y}$, Eq. (7) has infinitely many solutions for imaginary $\beta=i k_{n}$ corresponding to the bulk modes, and at most one solution for $\beta=\kappa \in \mathbb{R}$ corresponding to an edge mode that falls off exponentially fast away from the edge. It follows

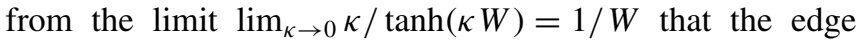

states only exist for $k_{y} \geqslant 1 / W$. This momentum cutoff has an associated energy cutoff $\varepsilon_{\mathrm{cut}}=\hbar v_{\mathrm{F}} / W$.

The two types of solutions (bulk and edge modes) are shown in Fig. 2 as full lines in black and red, respectively. It is clear from the figure that the TB and Dirac methods to calculate the band structure give very similar energies in the vicinity of the $K$ point and that the analytically found edge states match almost perfectly with the edgy $(\lambda \approx 1)$ states in TB. From the analytical model we just determined the exact energy range where the edge states are found. Given the great agreement between the two approaches, in the following, where we want to distinguish between bulk and edge states, we use the energy cutoff $\varepsilon_{\text {cut }}$ from Dirac theory to classify the TB states as either bulklike or edgelike.

\section{Armchair edge termination}

As the termination of an armchair ribbon has a mix of $A$ and $B$ lattice sites, as depicted in Fig. 1, we demand that the sublattice wave function vanishes on both edges. This results in a mixing of $K$ and $K^{\prime}$ states [41], which together with the general form of the solution (6) ultimately yield plane-wave states of the form $e^{i k_{n} x}$ with $k_{n}$ given by [41]

$$
k_{n}=\frac{n \pi}{W}-\frac{4 \pi}{3 \sqrt{3} a_{0}}=\frac{2 \pi[3 n-2(N+1)]}{3 \sqrt{3} a_{0}(N+1)},
$$

with $n \in \mathbb{Z}$, and the corresponding eigenenergies $\tilde{\epsilon}_{n}=$ $s \sqrt{k_{y}^{2}+k_{n}^{2}}$. In the second equality we have expressed the width of the ribbon as $W=(N+1) a_{0} \sqrt{3} / 2$, where $N$ is the number of atomic rows. From this form it follows that every third ribbon, where $3 n-2(N+1)=0$ can be fulfilled, will be semimetallic while the rest will have a band gap.

\section{B. Dimensionless scaling in Dirac theory}

An important property of the Dirac theory is a scale invariance of the ribbons: If all equations are rewritten in dimensionless units where the energies are scaled in units of the Fermi energy $\varepsilon_{\mathrm{F}}$, momenta in units of the Fermi momentum $k_{\mathrm{F}}$, and the distances with the ribbon width $W$, then one finds that the only system-dependent parameter is the dimensionless parameter $\Lambda \equiv k_{\mathrm{F}} W$. This insight is very useful, since it allows us to identify effects that should exist across all widths of ribbons, provided that their respective Fermi levels are scaled accordingly and of course that the Dirac model is valid.

In dimensionless form, the governing equations for the $\mathrm{ZZ}$ ribbons thus become

Bulk:

$$
\begin{aligned}
K_{y} & =\frac{K_{n}}{\tan \left(K_{n} \Lambda\right)}, \quad K_{y}=\frac{K}{\tanh (K \Lambda)} \\
E_{n} & =\sqrt{K_{y}^{2}+K_{n}^{2}}, \quad E_{e}=\sqrt{K_{y}^{2}-K^{2}} \\
\psi(\tilde{x}) & =C_{b} e^{i K_{y} \Lambda \tilde{y}}\left(\begin{array}{c}
i s \sin \left(\tilde{x} \Lambda K_{n}\right) \\
\sin \left([1-\tilde{x}] \Lambda K_{n}\right)
\end{array}\right) \\
\phi(\tilde{x}) & =C_{e} e^{i K_{y} \Lambda \tilde{y}}\left(\begin{array}{c}
i s \sinh (\tilde{x} \Lambda K) \\
\sinh ([1-\tilde{x}] \Lambda K)^{\prime}
\end{array}\right)
\end{aligned}
$$



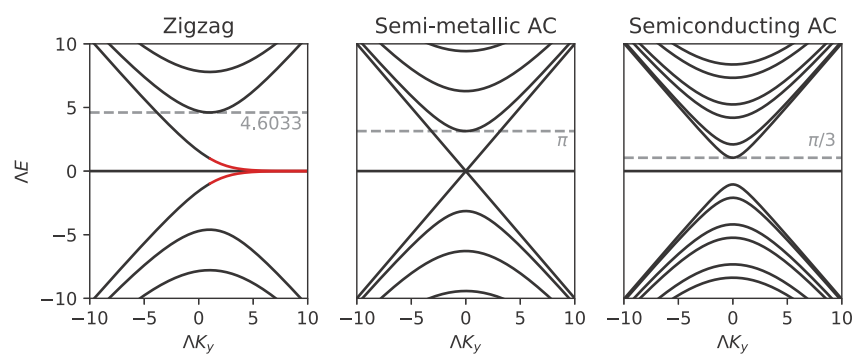

FIG. 3. The band structures of zigzag, semimetallic, and semiconducting ribbons in dimensionless units. We expect nonclassical behavior when the Fermi energy is close to or below the bottom parabolic band indicated with the gray dashed lines.

with dimensionless momentum $K_{y} \equiv k_{y} / k_{\mathrm{F}}$. The corresponding dimensionless momenta and energies of the bulk modes $\psi(\tilde{x})$ are denoted by $K_{n}$ and $E_{n} \equiv \epsilon_{n} / \varepsilon_{\mathrm{F}}$, those for the edge modes $\phi(\tilde{x})$ are called $K$ and $E_{e}$, and $\tilde{x}=x / W$ is the dimensionless lateral position in the ribbon. For the $\mathrm{AC}$ ribbons we find that

$$
K_{n}=\frac{\pi[3 n-2(N+1)]}{3 \Lambda} .
$$

Plots of the dimensionless band structures for the three different cases, zigzag, semimetallic, and semiconducting armchair ribbons are shown in Fig. 3. We emphasize the large differences between the band structures in the low-energy regime; especially the different placement of the bottom of the lowest parabolic band, to which we will return in the following.

One important consequence is that the scaling behavior of the electronic states will carry over to the plasmonic energies as well. As the band structure in the Dirac model is approximated with an infinite cone the (dimensionless) response will only depend on $\Lambda$, rather than on $\varepsilon_{F}$ and $W$ separately. This scale invariance holds, both when leaving out the edge states and when including them. The dimensionless form is used throughout the paper and thus we concern ourselves with the scaled plasmon energies given by $\hbar \omega_{p} / \varepsilon_{\mathrm{F}}$.

Given the great agreement in the band structures of the numerical tight-binding and the analytical Dirac models, we expect that also for TB calculations there will be parameters for which the scaled plasmonic energies will be scale invariant for constant values of the parameter $\Lambda$, even though the scale invariance does not strictly hold in the TB model.

\section{Emergence of nonclassicality}

The quantum mechanical Dirac model for ribbons has a classical limit, and vice versa away from the classical limit we will identify the onset of nonclassical behavior. When there are many bands crossing the Fermi energy one would expect the system to behave classically. On the other hand, for combinations of widths and Fermi energies where the Fermi surface is only crossed by a few states we are starting to probe the quantumness of the system and expect deviations from the classical regime. As our heuristic measure, we take the bottom energy of the lowest parabolic band as the separation between the quantum and classical regimes. Interestingly, from Fig. 3 this value differs for $\mathrm{ZZ}$ and $\mathrm{AC}$ ribbons, and it differs also for the semimetallic and the semiconducting AC ribbons. These different critical values $\Lambda_{c}$ at which we predict the classicalto-quantum behavior to occur can be determined analytically (and further below we will test them against numerical TB calculations).

a. For zigzag. By setting to zero the derivative of the energy with respect to the dimensionless momentum $K_{y}$, it is found that the sought bottom of the band occurs at $K_{y}=1 / \Lambda$, corresponding to $k_{y}=1 / W$. By inserting this into the scaled expression for the band energies, we find that

$$
E_{n}=\sqrt{\Lambda^{-2}+K_{n}^{2}\left(\Lambda^{-1}\right)}=\frac{\sqrt{1+\xi_{n}^{2}}}{\Lambda},
$$

where $\Lambda K_{n}=\xi_{n}=\tan \left(\xi_{n}\right)$. Looking for the solution where the Fermi energy crosses the bottom parabolic band, i.e., $\epsilon_{n} / \varepsilon_{\mathrm{F}}=E_{n}=1$, it is found that the critical value is

$$
\Lambda_{\mathrm{c}}^{\mathrm{zz}}=\sqrt{1+\xi_{1}^{2}} \approx 4.6033 .
$$

This $\Lambda_{\mathrm{c}}^{\mathrm{zz}}$ is a dimensionless number, and with this single number we predict with Dirac theory the emergence of quantum effects both in narrow ribbons at high Fermi levels and in wide ribbons with low doping. As we will see below, this is indeed the value around which the dipole plasmon energies start to deviate from the classical results for zigzag ribbons.

b. For armchair. For ribbons with armchair edge terminations, in the limit of many atoms, the band bottoms occur at $E_{n}= \pm n \pi / \Lambda$ for the semimetallic ribbons and at $E_{n} \in$ $\{ \pm(3 n+1) \pi / 3 \Lambda, \pm(3 n+2) \pi / 3 \Lambda\}$ for the semiconducting ribbons, with $n \in \mathbb{N}$. That is,

$$
\Lambda_{\mathrm{c}}^{\mathrm{ac}}=\left\{\begin{array}{ll}
\pi & \text { for semimetallic AC ribbons } \\
\frac{\pi}{3} & \text { for semiconducting AC ribbons }
\end{array} .\right.
$$

Unlike for the $\mathrm{ZZ}$ ribbons, the band structures for AC ribbons are symmetric around the Dirac points and in that sense they are thus more like the bulk graphene bands. Combined with the lower value of $\Lambda_{\mathrm{c}}^{\mathrm{ac}}$, we expect classical behavior down to smaller values of $\Lambda$ for armchair ribbons.

\section{CLASSICAL PLASMONS}

It is naturally also possible to calculate the plasmons classically. For the ribbon geometry this has already been done in different ways $[7,26,46,47]$. When combined with the continuity equation, the coupling between the potential $\phi(\mathbf{r})$ and the induced charge density $\rho(\mathbf{r})$ can be written as an integrodifferential eigensystem of equations as

$$
\begin{aligned}
\zeta_{n} \phi_{n}(\mathbf{r}) & =\frac{-1}{2 \pi} \int d^{2} \mathbf{r} \frac{\nabla^{\prime} \cdot\left[f\left(\mathbf{r}^{\prime}\right) \nabla^{\prime} \phi_{n}\left(\mathbf{r}^{\prime}\right)\right]}{\left|\mathbf{r}-\mathbf{r}^{\prime}\right|}, \\
\zeta_{n} & =\frac{2 i \epsilon_{0} \omega_{n} W}{\sigma\left(\omega_{n}\right)},
\end{aligned}
$$

where all coordinates and differential operators work in the 2D plane of the graphene. The graphene is treated as being embedded in an $\epsilon=1$ material. It has here been assumed that the conductivity is uniform inside the ribbon of width $W$, and 
vanishes outside:

$$
\begin{aligned}
\sigma(\mathbf{r}, \omega) & =\sigma(\omega) f(\mathbf{r}), \quad \text { with } \\
f(\mathbf{r}) & = \begin{cases}1 & \text { for } \mathbf{r} \text { inside the ribbon, } \\
0 & \text { for } \mathbf{r} \text { outside the ribbon. }\end{cases}
\end{aligned}
$$

High-precision fits of the values of the eigenvalues $\zeta_{n}$ in Eq. (14) are given by Christensen (Ref. [46]) for the first seven modes. We have used these values in our classical calculations together with the low-temperature, local conductivity $\sigma(\omega)$ for bulk graphene. This conductivity can be derived, among other ways, from the Dirac model in the limit of infinitely wide ribbons or from the general expression of the bulk polarizability of graphene as found by Hwang and Das Sarma, [48] and by Wunsch et al. [43]. Here we just present the resulting expressions for the intraband and the interband contributions that together make up $\sigma(\omega)$ :

$$
\begin{aligned}
\sigma_{\text {intra }}(\omega) & =\frac{i e^{2} \varepsilon_{\mathrm{F}}}{\pi \hbar^{2}(\omega+i \eta)}, \\
\sigma_{\text {inter }}(\omega) & =\frac{e^{2}}{4 \hbar}\left[\frac{i}{\pi} \ln \left|\frac{2 \varepsilon_{\mathrm{F}}-\hbar \omega}{2 \varepsilon_{\mathrm{F}}+\hbar \omega}\right|+\Theta\left(\hbar \omega-2 \varepsilon_{\mathrm{F}}\right)\right],
\end{aligned}
$$

where $\Theta$ is the Heaviside step function. By combining Eqs. (14) and (15) we can find the plasma energies as a function of the ribbon width.

For our purposes it is essential to realize that Eq. (14b) can be rewritten in dimensionless variables as $\sigma\left(v_{n}\right) \zeta_{n}=$ $2 i \epsilon_{0} \hbar v_{\mathrm{F}} v_{n} \Lambda$, with the dimensionless plasmon energy $v_{n}=$ $\hbar \omega_{n} / \varepsilon_{\mathrm{F}}$ and again $\Lambda=k_{\mathrm{F}} W$. This insight turns out to be quite practical, because it is sufficient to calculate the connection between $v_{n}$ and $\Lambda$ only once to obtain the plasmon energies for all combinations of widths and Fermi momenta that satisfy $\Lambda=k_{\mathrm{F}} W$. Moreover, in Sec. III B we saw that the Dirac model has the same scale invariance. So we find that the scaling property holds both inside and outside the classical regime, as long as Dirac theory is accurate. We will test the latter by comparing Dirac and classical theories with tightbinding calculations in the next section.

Let us summarize our models and explain our terminology. The classical model is a local continuum model that assumes that the conductivity of the nanoribbon is a uniform material parameter equal to the sheet conductivity of infinite graphene. For simplicity we call this model classical even though the value for the sheet conductivity by Eq. (15) depends on $\hbar$, which would be an argument for calling the model semiclassical instead. As a next level of modeling one could adopt a hydrodynamic continuum model (not done here) in which the response becomes nonlocal $[17,46]$, and the conductivity would depend on two spatial coordinates. Hydrodynamic models are often classified as semiclassical [49]. Then comes the Dirac model, which is quantum mechanical in the sense that the bands are quantized and accounted for individually in the evaluation of the dielectric function. It incorporates some atomistic details via the boundary conditions and will thus provide different results for $\mathrm{AC}$ and $\mathrm{ZZ}$ edge terminations [17]. Finally, the TB calculations are fully quantum as all atoms and bands individually add to the evaluation of the plasmons.

\section{NUMERICAL AND ANALYTICAL RESULTS COMPARED}

We present two comparisons: quantum versus classical plasmons in Sec. V A, and properties of atomistic (TB) quantum plasmons versus those of continuum (Dirac) quantum plasmons in Sec. V B.

\section{A. Quantum versus classical plasmons}

Here we systematically investigate the range of validity of the classical description for graphene ribbons, by comparing with TB quantum calculations. In particular, we will test the heuristic value of the various $\Lambda_{c}$ that we identified in Sec. III C for characterizing the emergence of nonclassical behavior in a scale invariant way. In Sec. II B we outlined how one can identify quantum plasmons of nanostructures within a tight-binding formalism, and here we apply this approach to graphene ribbons. The calculation of the corresponding classical plasmons was described in Sec. IV.

Guided by the scaling properties of the Dirac and classical models, in Fig. 4 we present the plasmonic energies as a function of the dimensionless variable $\Lambda$. The figure shows a comparison of the scaled plasmonic energy as calculated with the TB model of Sec. II A and in the classical model for both $\mathrm{ZZ}$ and $\mathrm{AC}$ ribbons, and when considering only the intraband contribution (top panels of Fig. 4) or all transitions (bottom panels). By "intraband" we mean that we only include eigenstates with energies above the cutoff energy $\varepsilon_{\text {cut }}=\hbar v_{\mathrm{F}} / W$ for the edge states for zigzag ribbons and above zero energy for armchair ribbons, which corresponds to only considering intraband transitions in a classical, wide-ribbon limit. For ribbons of finite widths, the transitions are intraband transitions in the sense that the bands in the upper cone are size-constriction foldings of the infinite graphene conduction band, although the actual transitions do occur between bands of the ribbon.

We see that for large values of $\Lambda$, the classical and all TB calculations agree across all four panels. There is no visible effect of either edge terminations or other quantum effects there. Furthermore, the TB calculations for different Fermi levels agree very well as predicted from the scaling of the Dirac model. For smaller values of $\Lambda$ the plasmon energies as calculated by the TB model start to depart from the classical values.

For zigzag ribbons, Fig. 4(a) constitutes a confirmation of our prediction in Eq. (12) that this onset of quantum behavior occurs at $\Lambda_{\mathrm{c}} \simeq 4.6$, the point at which the lowest of the parabolic bands of the zigzag ribbons crosses the Fermi level. This same onset is seen both in the "Drude-like" case [Fig. 4(a), top panel] and with all transition included [Fig. 4(a), bottom panel].

Another important feature of Fig. 4(a) is that the tightbinding plasmon energies for $\varepsilon_{F}=0.4 \mathrm{eV}$ and $0.8 \mathrm{eV}$ are indeed quite close to each other in the chosen dimensionless units, and closer to each other than to the classical plasmon curves. Dirac theory predicts that the two quantum plasmon calculations would coincide exactly, and the tight-binding calculations confirm that the scale invariance of Dirac theory indeed holds approximately. A better overview and insight when 

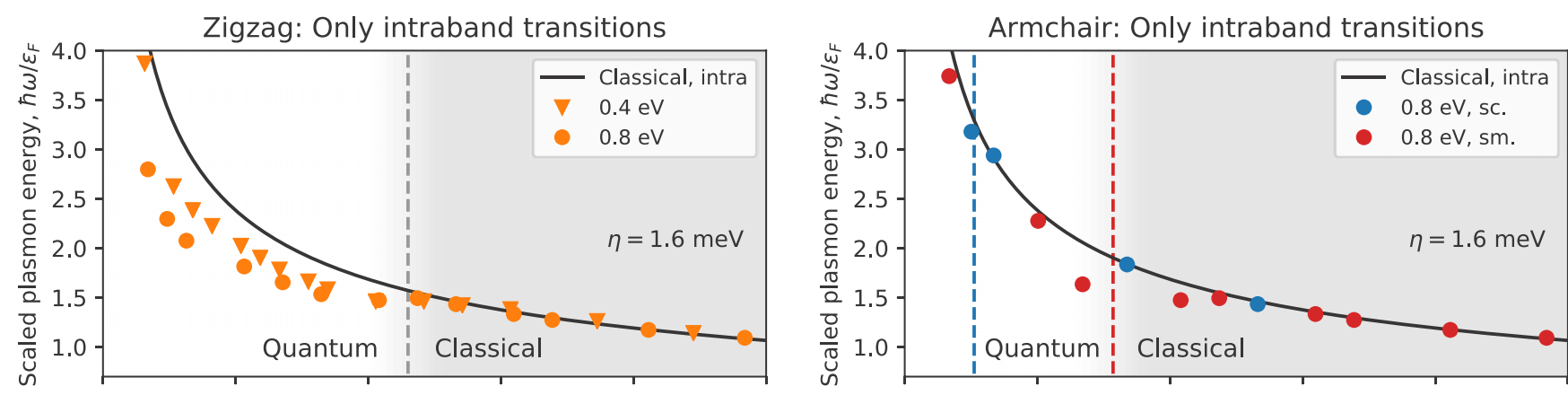

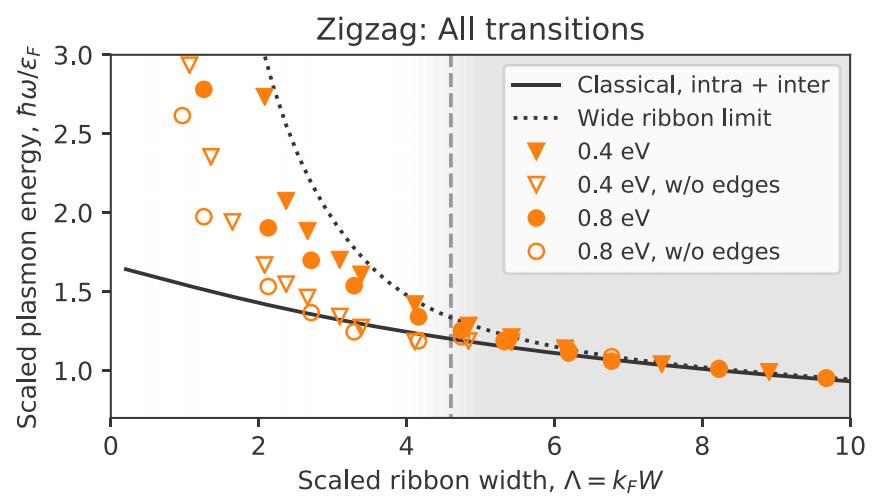

(a) Zigzag

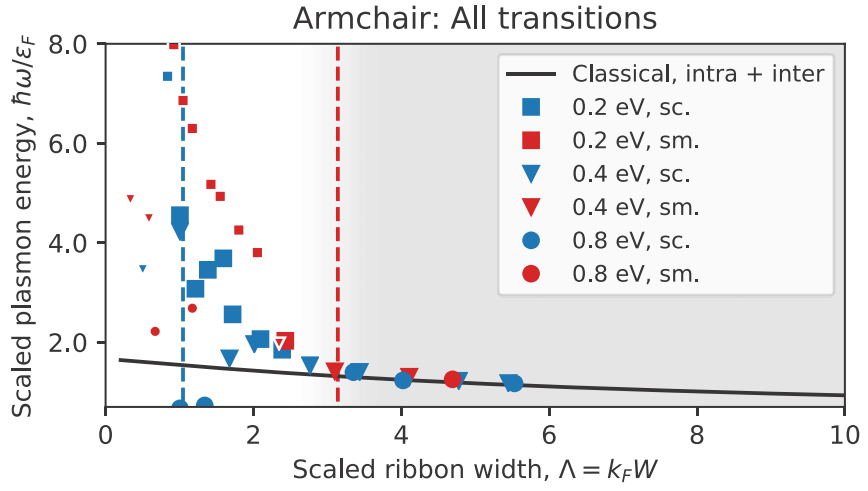

(b) Armchair

FIG. 4. Scaled plasmon energy as a function scaled ribbon width $\Lambda$. The value $\Lambda=5$ corresponds for example to a width of $16.4 \mathrm{~nm}$ for $\varepsilon_{\mathrm{F}}=0.2 \mathrm{eV}$. (a) $\Lambda$ is varied by changing $W$ while keeping the Fermi energy fixed at either $0.4 \mathrm{eV}$ (triangles) or $0.8 \mathrm{eV}$ (dots). The vertical dashed line corresponds to $\Lambda_{\mathrm{c}}^{\mathrm{zz}} \simeq 4$.6. The open symbols in the bottom panel correspond to calculations of plasmon energies where edge states were removed from the calculation. (b) Top: The intraband plasmons of AC ribbons, both the classical prediction and the quantum plasmon predictions for semiconducting and for semimetal ribbons. Blue and red dashed vertical lines correspond to $\Lambda_{\mathrm{c}}^{a c}=\pi / 3$ and $\pi$, respectively. (b) Bottom: As in the top panel, but now including all transitions. The small symbols are used for the peaks in the loss spectrum that are not associated with an actual plasmon defined as $\operatorname{Re}\left(\epsilon_{n}\right)=0$.

scale invariance holds in TB calculations will be presented in Sec. V B below. The dotted line in the bottom panel shows the interpolated data from calculations of a $9 \mathrm{~nm}$ wide ribbon at varying Fermi energy and provides the best guess, given the calculations that have been done, of the behavior of arbitrarily wide ribbons where the plasmon energies have converged with respect to the number of bands. This will be explored further in the following section. Comparing the results for the $\varepsilon_{\mathrm{F}}=0.4 \mathrm{eV}$ and $\varepsilon_{\mathrm{F}}=0.8 \mathrm{eV}$ ribbons we see that lowering the Fermi energy, which for constant $\Lambda$ corresponds to widening the ribbons, moves the points closer to the dotted line, as expected.

By excluding the zigzag edge states in the evaluation of $\chi^{0}$ open symbols in the bottom panel of Fig. 4(a), we find a significant plasmon red shift in the quantum regime. In other words, edge states of zigzag nanoribbons contribute with a significant blue shift of the plasmon energies in the quantum regime, while they have hardly any impact on the energy in the classical regime above $\Lambda_{\mathrm{c}}^{\mathrm{zz}}$. This effect of edge states becomes even more evident by directly plotting the energy shift as in Fig. 5. Clearly, for zigzag ribbons the edge states do not affect the plasmon energies for $\Lambda>\Lambda_{\mathrm{c}}^{\mathrm{zZ}}$ and give rise to a blue shift for $\Lambda<\Lambda_{\mathrm{c}}^{\mathrm{zZ}}$. The found blue shift is in stark contrast to the results for graphene disks [17] and triangles [18] in which the zigzag edge states are found to give rise to a net red shift of the plasmon energies. Back to our ribbons, for $\Lambda<1$ the Fermi level crosses the edge state and the evaluation of the edge-state contribution in the manner described above becomes meaningless.

Having discussed quantum-classical transitions for zigzag ribbons, we now return to Fig. 4 and study armchair ribbons in Fig. 4(b). The picture is slightly different for armchair ribbons as they exist as either semiconducting (sc.) or semimetallic (sm.). When including only intraband transitions, the scaled plasmon energies follow the classical behavior rather closely

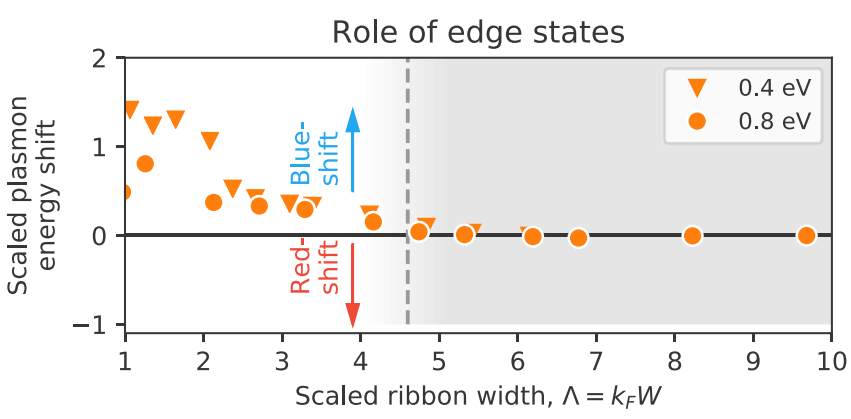

FIG. 5. Scaled plasmon energies in the presence of edge states minus scaled plasmon energies when neglecting the edge states, as a function of the scaled ribbon width $\Lambda$, for two fixed values of the Fermi energy. 

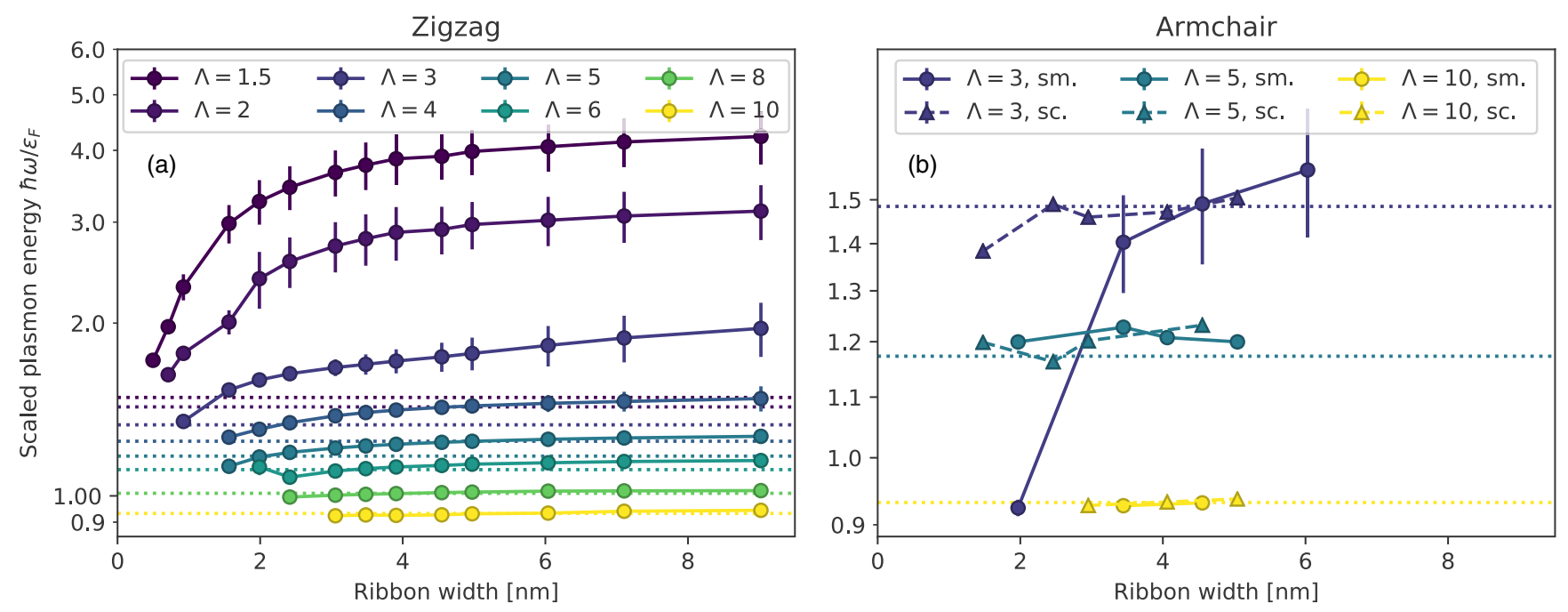

FIG. 6. The plasmon energy scaled with the Fermi energy for constant $\Lambda$ at varying ribbon widths, for both (a) ZZ and (b) AC ribbons. Dotted horizontal lines in equal colors are the corresponding classical plasmon energies. In the AC panel the data is split into semimetallic (sm.) and semiconducting (sc.) ribbons. This distinction is only important for the low $\Lambda$ structures, as seen. The bars show the width of the plasmon peak in the loss spectrum. All displayed data points have $\varepsilon_{\mathrm{F}}<2.0 \mathrm{eV}$.

across the entire range, except for a single outlier. As discussed above, because of the symmetry around the $K$ point of the armchair band structure, we do not expect the same kind of quantum-classical transition as for zigzag ribbons. In the bottom panel of Fig. 4(b) we have split the ribbons into the two types. The vertical, dashed lines indicate the position of the band bottom in the appropriate color. As expected, the deviation from classical results starts at lower $\Lambda$ than previously for the zigzag ribbons. The small symbols in the bottom panel of Fig. 4(b) denote peaks in the loss spectrum that are not associated with real plasmons as there is no simultaneous crossing of the real part of the dielectric eigenvalues with zero. For the semiconducting ribbons the plasmon cease to exist when the Fermi energy crosses the lowest parabolic band at $\Lambda=\pi / 3$. For the semimetallic ribbons the plasmons cease to exist earlier, namely already below $\Lambda=\pi$. There seems to be an exception with the red square just above $\Lambda=2$ (which lies beneath a small, red triangle), but as the TB calculations are done for room temperature $k_{b} T \approx 25 \mathrm{meV}$, there will still be a finite population of electrons in the bottom parabolic band for this point. For the smallest values of $\Lambda$ for which plasmons still exist, the positions of the main dipole plasmon peaks become increasingly hard to locate, resulting in an increased scatter of the data points, as also reported elsewhere $[17,26]$.

\section{B. Emergent scale invariance for plasmons}

In general, the tight-binding model for graphene ribbons does not have the same scale invariance that we found both for Dirac theory and for classical plasmonics, as the TB band structure does not consist of an infinite Dirac cone. This follows from the fact that, due to the infinite cone shape, the band structures for two different ribbon widths in the Dirac description are related be a simple scaling transformation while this is not the case for the more complex TB band structure. But since the low-energy bands calculated with TB and with Dirac theory agree so well, at least for the parameters of Fig. 2, the scale invariance will be an emergent property of the TB model, valid only in part of the parameter space spanned by $\left\{\varepsilon_{\mathrm{F}}, W\right\}$. Only in that subspace can classical and/or Dirac theory be expected to agree with TB calculations.

As a test of the proposed scale invariance we conduct a range of calculations where $\Lambda$ is held constant while the widths of the ribbons are varied, so doubling the size of the ribbon goes hand in hand with halving the Fermi energy. As previously stated, we expect the scaled plasmon energy to tend towards a constant when the ribbons get wider and the Dirac model becomes a better description. It is less clear how fast the limit will be reached. When the Fermi energy is above $2.0 \mathrm{eV}$ we are well out of the linear regime of the bands and do not expect the Dirac scaling to work anymore. For the armchair ribbons we distinguish between semiconducting and semimetallic ones, as this should have an impact for small values of $\Lambda$ where the Fermi energy is close to the difference in the band structures.

As one of our main results we present in Fig. 6 how the TB plasmon energies converge as ribbon widths are increased. For $\Lambda \gg 1$ the plasmon energies quickly converge for larger widths to a value that differs little from the classical plasmon energy. But it is important to notice that the wide-ribbon limits in this figure do not automatically coincide with the classical limit, as one might expect: for $\Lambda$ not much larger than unity, there is a clear discrepancy between the converged energies of the TB plasmons and the classical plasmons. Wherever the TB curves in Fig. 6 have become (almost) horizontal, the scale invariance that holds exactly for Dirac and classical plasmons has also emerged for TB quantum plasmons.

The bending of the curves for smaller widths illustrates the shortcomings of the scalability of the Dirac model: For it to hold exactly, we would need infinitely many bands in the band structure, but as the number of atoms in the full-width supercell decreases as $W$ is reduced (recall Fig. 1), we will get fewer bands instead and thus a deviation from the converged constant plasmon energy as obtained for wide ribbons. In 


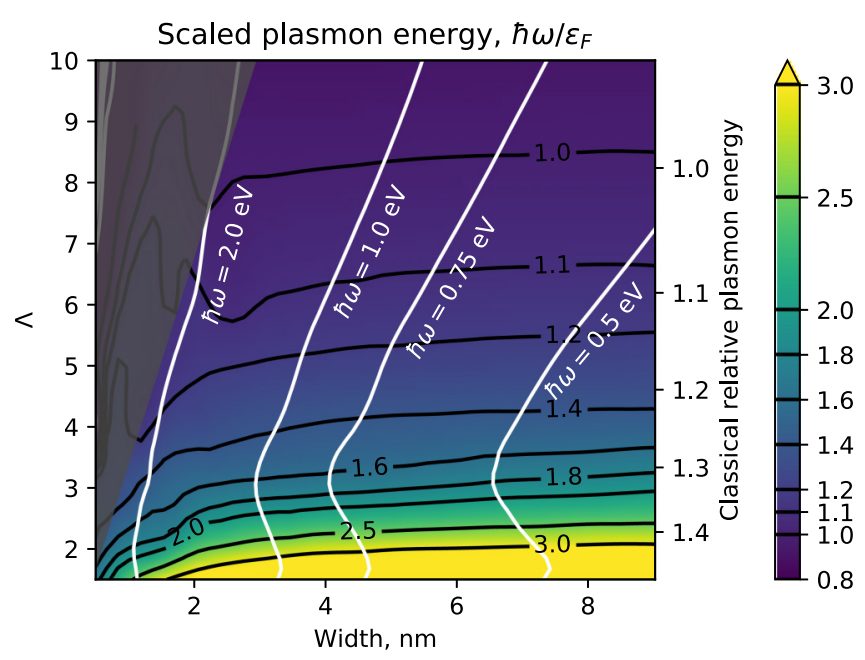

FIG. 7. The scaled plasmon energy as a function of the width and $\Lambda$ for zigzag ribbons. The white lines indicate paths with constant (absolute) plasmon energy. The gray area corresponds to structures with $\varepsilon_{\mathrm{F}}>2 \mathrm{eV}$ where we expect to be outside the linear regime of the bands. For larger widths the contours start to converge as expected from Dirac theory. The departure from horizontal lines is a signature of having only a finite number of electronic states. The right $y$ axis shows the classical results for comparison.

Fig. 6 we also see that the AC plasmon energies in general are closer to the classical predictions than the $\mathrm{ZZ}$ plasmon energies, as could also be extracted from Fig. 4.

In Fig. 7 we display the same data for zigzag ribbons as in Fig. 6 but in a complementary way, now as a function of width and $\Lambda$. We obtain a surface plot of the scaled plasmon energy, where data points have been cubicly interpolated to get a smooth surface. From this view, we also see the convergence of the scaled plasmon energies that deviates significantly for the classical values displayed on the right $y$ axis. For $\varepsilon_{\mathrm{F}}>$ $2.0 \mathrm{eV}$ the Fermi energy is outside the linear part of the band structure and we find large deviations from the Dirac model as can be seen in the gray area of the plot. By multiplying with the corresponding Fermi energy surface $\varepsilon_{\mathrm{F}}=\hbar v_{\mathrm{F}} \Lambda / W$ we calculate lines of constant plasmon energy (the white lines) thus conveniently providing a continuous range of options in parameter space to obtain a specific plasmon energy. The bending of the white lines also reveals the offset from the classical behavior: For large values of $\Lambda$ the system is well described when only including the classical intraband Drude term. This results in a scaling of the scaled plasmon energy with $\Lambda^{-1 / 2}$ leading to the plasmon energy scaling as $\hbar \omega \propto$ $\Lambda^{1 / 2}$. The intraband divergence at $\Lambda=0$ is quenched due to the screening of the interband transitions when the latter are included. Looking at the data in Fig. 7, for a constant width, when going to smaller values of $\Lambda$, around $\Lambda_{c}$ the plasmon energies start to increase again showing that the scaled plasmon energy must increase faster than as $\Lambda^{-1}$.

\section{CONCLUSIONS}

Using tight-binding calculations and inspired by Dirac theory, we identify multiple interesting effects in graphene nanoribbon plasmons: As a first main result, for both armchair and zigzag ribbons an emerging nonclassical scale-invariant behavior of the plasmon energies has been predicted and confirmed to exist also in TB calculations, even though the scale invariance does not hold strictly in the mathematical sense in TB. For ribbons wider than $5 \mathrm{~nm}$, we illustrated in Fig. 6 that the scale invariance effectively holds for the energies considered, and better so for higher Fermi energy (which we kept smaller than $2.0 \mathrm{eV}$ ). The scale-invariant horizontal curves that the TB calculations converge to generally have nonclassical limiting values. This is the realm where Dirac theory can be accurate, and only in the limit $\Lambda \equiv k_{F} W \gg$ 1 do our TB plasmon energies agree nicely with those of classical plasmons.

An experimental realization that comes close to the nonclassical regime is the work in Ref. [40] where the plasmonic mode of a $15 \mathrm{~nm}$ ribbon with $\varepsilon_{\mathrm{F}} \approx 0.4 \mathrm{eV}$ has been measured. This is still within the classical regime as $\Lambda \approx 9.3>\Lambda_{\mathrm{c}}$, but lowering the Fermi energy to around $0.1 \mathrm{eV}$ corresponding to $\Lambda \approx 2.3$ should reveal new quantum effects for both zigzag and armchair edge terminations.

As our second main result, we have related the energy of the bottom parabolic band at the $K$ points to the onset of the deviation from the classical model and calculated these energies analytically using the Dirac model. Here again, we find that the agreement between our heuristic analytical estimates and numerical calculations holds quite well and in a scale-invariant way, i.e., the analytical estimates describe the onset of nonclassical plasmonics both for narrow ribbons with higher Fermi energies and wider ribbons with lower $\varepsilon_{\mathrm{F}}$. Spectral differences between quantum and classical plasmons emerge slightly earlier for zigzag than for armchair ribbons (i.e., for larger $\Lambda$, or already for wider ribbons at equal Fermi energy).

Third, for armchair nanoribbons we observe the disappearance of the plasmons at two different low values of the scaled ribbon width $\Lambda$, dependent on whether the ribbons are semiconducting or semimetallic in their neutral state. Fourth, for zigzag ribbons we have provided a convenient way of predicting absolute plasmon energies from the isofrequency curves in Fig. 7. Finally, we revealed how the edge states of nanoribbons contribute with a significant blue shift of plasmon energies, in contrast to reported red shifts for other graphene nanostructures.

\section{ACKNOWLEDGMENTS}

We would like to thank Thomas Christensen, Johan R. Maack, and P. André D. Gonçalves for stimulating discussions. This work was supported by the Danish Council for Independent Research-Natural Sciences (Project No. 1323-00087). The Center for Nanostructured Graphene is sponsored by the Danish National Research Foundation (Project No. DNRF103). N.A.M. is supported by VILLUM FONDEN (Grant No. 16498). K.S.T. acknowledges funding from the European Research Council (ERC) under the European Union's Horizon 2020 Research and Innovation Program (Grant Agreement No. 773122, LIMA). 


\section{APPENDIX A: IDENTIFYING EDGE STATES IN TIGHT-BINDING}

In the Dirac model the edge states of graphene zigzag ribbons are readily found as solutions that decay exponentially fast from the edge of the structure in contrast to the bulklike modes that behave more like standing waves. As the tight-binding model is solved numerically by diagonalizing the Hamiltonian we do not get this distinction for free, but need to analyze the resulting states subsequently in order to classify them properly. To give an overview of where in the band diagrams calculated in TB we find these edge states, we introduce an operational definition of edginess as

$$
\lambda_{n}(k)=\frac{\sum_{l \in \Omega}\left|\psi_{n l}(k)\right|^{2}-\sum_{l \notin \Omega}\left|\psi_{n l}(k)\right|^{2}}{\sum_{l}\left|\psi_{n l}(k)\right|^{2}},
$$

where $l$ refers to the atomic sites. In other words, the edginess $\lambda_{n}$ of the $n$th state is found by the amount of the wave function localized on the edge of the ribbon, $\Omega$, subtracted with the weight in the middle of the ribbon. In our case we define $\Omega$ as the outermost quarters of the atoms on either side of the ribbon. Using this definition, an edge mode will have $\lambda \simeq 1$ while eigenstates located entirely in the center of the ribbon will have $\lambda=-1$.

\section{APPENDIX B: COULOMB INTERACTION IN REAL SPACE}

At large distances, the Coulomb interaction between two sites will be predominantly pointlike and thus scale as their inverse distance. This long-range $r^{-1}$ behavior makes it impossible to calculate the correct Coulomb interaction term in real space due to lack of convergence. Fortunately, this is not necessary either, as we ultimately are interested in the dielectric function. That is, we only need the correct $V \chi^{0}$ product, while not necessarily the correct form of the $V$ matrix itself (which is ill defined). So, as we require charge neutrality, we utilize that $\sum_{i} \chi_{i j}^{0}=0$ to calculate a modified interaction [26]

$$
\tilde{V}_{i j}=\sum_{n, j}\left(V_{i 0, j n}-|n b|^{-1}\right),
$$

which fulfills $V \chi^{0}=\tilde{V} \chi^{0}$ and falls off more quickly with distance than $V$. We have used the notation $V_{i 0, j n}$ to mean the interaction between the $i$ th site in the zeroth supercell and the $j$ th site in supercell $n$. It is important to stress that by this approach we do not screen the Coulomb interaction in a physical sense. Rather, it is a computational trick that allows us to evaluate the two well-defined matrices $\tilde{V}$ and $\chi^{0}$ individually and to obtain the correct dielectric function from their product. Alternatively, one could have converged $\sum_{n, l} V_{i 0, n l} \chi_{l j}^{0}$ directly with respect to $n$.

In the short-distance limit, keeping the assumption of pointlike interactions would lead to a diverging Coulomb term for for the distance going to zero. Instead, for sites close to each other and, ultimately, for a site interacting with itself, the spatial extent of the $p_{z}$ orbitals should be taken into account. This has been done by Ref. [26] and we adopt the same approach for all distances (with data acquired through private correspondence between our groups), whereby the Coulomb term no longer diverges for vanishing distances.
[1] K. S. Novoselov, A. K. Geim, S. V. Morozov, D. Jiang, Y. Zhang, S. V. Dubonos, I. V. Grigorieva, and A. A. Firsov, Electric field effect in atomically thin carbon films, Science 306, 666 (2004).

[2] K. S. Novoselov, V. I. Fal'ko, L. Colombo, P. R. Gellert, M. G. Schwab, and K. Kim, A roadmap for graphene, Nature (London) 490, 192 (2012).

[3] F. J. García de Abajo, Graphene plasmonics: challenges and opportunities, ACS Photonics 1, 135 (2014).

[4] F. Bonaccorso, Z. Sun, T. Hasan, and A. C. Ferrari, Graphene photonics and optoelectronics, Nat. Photon. 4, 611 (2010).

[5] A. N. Grigorenko, M. Polini, and K. S. Novoselov, Graphene plasmonics, Nat. Photon. 6, 749 (2012).

[6] S. Xiao, X. Zhu, B.-H. Li, and N. A. Mortensen, Grapheneplasmon polaritons: From fundamental properties to potential applications, Front. Phys. 11, 117801 (2016).

[7] P. A. D. Gonçalves and N. M. R. Peres, An Introduction to Graphene Plasmonics (World Scientific, Singapore, 2016).

[8] T. Low and P. Avouris, Graphene plasmonics for terahertz to mid-infrared applications, ACS Nano 8, 1086 (2014).

[9] T. Stauber, Plasmonics in Dirac systems: from graphene to topological insulators., J. Phys.: Condens. Matter 26, 123201 (2014).

[10] K. F. Mak and J. Shan, Photonics and optoelectronics of 2D semiconductor transition metal dichalcogenides, Nat. Photon. 10, 216 (2016).
[11] J. Cai, P. Ruffieux, R. Jaafar, M. Bieri, T. Braun, S. Blankenburg, M. Muoth, A. P. Seitsonen, M. Saleh, X. Feng, K. Müllen, and R. Fasel, Atomically precise bottom-up fabrication of graphene nanoribbons, Nature (London) 466, 470 (2010).

[12] A. Narita, X. Feng, Y. Hernandez, S. A. Jensen, M. Bonn, H. Yang, I. A. Verzhbitskiy, C. Casiraghi, M. R. Hansen, A. H. Koch, G. Fytas, O. Ivasenko, B. Li, K. S. Mali, T. Balandina, S. Mahesh, S. De Feyter, and K. Müllen, Synthesis of structurally well-defined and liquid-phase-processable graphene nanoribbons, Nat. Chem. 6, 126 (2014).

[13] A. Narita, X.-Y. Wang, X. Feng, and K. Müllen, New advances in nanographene chemistry, Chem. Soc. Rev. 44, 6616 (2015).

[14] P. Ruffieux, S. Wang, B. Yang, C. Sanchez-Sanchez, J. Liu, T. Dienel, L. Talirz, P. Shinde, C. A. Pignedoli, D. Passerone, T. Dumslaff, X. Feng, K. Müllen, and R. Fasel, On-surface synthesis of graphene nanoribbons with zigzag edge topology, Nature (London) 531, 489 (2016).

[15] S. Wang, L. Talirz, C. A. Pignedoli, X. Feng, K. Müllen, R. Fasel, and P. Ruffieux, Giant edge state splitting at atomically precise graphene zigzag edges, Nat. Commun. 7, 11507 (2016).

[16] S. Thongrattanasiri and F. J. García de Abajo, Optical Field Enhancement by Strong Plasmon Interaction in Graphene Nanostructures, Phys. Rev. Lett. 110, 187401 (2013).

[17] T. Christensen, W. Wang, A. P. Jauho, M. Wubs, and N. A. Mortensen, Classical and quantum plasmonics in graphene 
nanodisks: Role of edge states, Phys. Rev. B 90, 241414(R) (2014)

[18] W. Wang, T. Christensen, A.-P. Jauho, K. S. Thygesen, M. Wubs, and N. A. Mortensen, Plasmonic eigenmodes in individual and bow-tie graphene nanotriangles, Sci. Rep. 5, 9535 (2015).

[19] M. Settnes, J. R. M. Saavedra, K. S. Thygesen, A.-P. Jauho, F. J. García de Abajo, and N. A. Mortensen, Strong plasmon-phonon splitting and hybridization in 2D materials revealed through a self-energy approach, ACS Photonics 4, 2908 (2017).

[20] Z. Wang, T. Li, K. Almdal, N. A. Mortensen, S. Xiao, and S. Ndoni, Experimental demonstration of graphene plasmons working close to the near-infrared window, Opt. Lett. 41, 5345 (2016).

[21] Z.-B. Zheng, J.-T. Li, T. Ma, H.-L. Fang, W.-C. Ren, J. Chen, J.-C. She, Y. Zhang, F. Liu, H.-J. Chen, S.-Z. Deng, and N.-S. Xu, Tailoring of electromagnetic field localizations by two-dimensional graphene nanostructures, Light Sci. Appl. 6, e17057 (2017).

[22] Y. Fan, Z. Liu, F. Zhang, Q. Zhao, Z. Wei, Q. Fu, J. Li, H. Gu, and C. Li, Tunable mid-infrared coherent perfect absorption in a graphene meta-surface, Sci. Rep. 5, 13956 (2015).

[23] Y. Fan, N.-H. Shen, T. Koschny, and C. M. Soukoulis, Tunable terahertz meta-surface with graphene cut-wires, ACS Photonics 2, 151 (2015)

[24] M. Zarenia, A. Chaves, G. A. Farias, and F. M. Peeters, Energy levels of triangular and hexagonal graphene quantum dots: A comparative study between the tight-binding and Dirac equation approach, Phys. Rev. B 84, 245403 (2011).

[25] C. Mansilla Wettstein, F. P. Bonafé, M. B. Oviedo, and C. G. Sánchez, Optical properties of graphene nanoflakes: Shape matters, J. Chem. Phys. 144, 224305 (2016).

[26] S. Thongrattanasiri, A. Manjavacas, and F. J. García de Abajo, Quantum finite-size effects in graphene plasmons, ACS Nano 6, 1766 (2012).

[27] F. Karimi and I. Knezevic, Plasmons in graphene nanoribbons, Phys. Rev. B 96, 125417 (2017).

[28] A. A. Shylau, S. M. Badalyan, F. M. Peeters, and A.-P. Jauho, Electron polarization function and plasmons in metallic armchair graphene nanoribbons, Phys. Rev. B 91, 205444 (2015).

[29] L. Brey and H. A. Fertig, Elementary electronic excitations in graphene nanoribbons, Phys. Rev. B 75, 125434 (2007).

[30] C. E. P. Villegas, M. R. S. Tavares, G.-Q. Hai, and P. Vasilopoulos, Plasmon modes and screening in double metallic armchair graphene nanoribbons, Phys. Rev. B 88, 165426 (2013).

[31] D. R. Andersen and H. Raza, Plasmon dispersion in semimetallic armchair graphene nanoribbons, Phys. Rev. B 85, 075425 (2012)

[32] T. Wenger, G. Viola, M. Fogelström, P. Tassin, and J. Kinaret, Optical signatures of nonlocal plasmons in graphene, Phys. Rev. B 94, 205419 (2016).

[33] P. A. D. Gonçalves, E. J. C. Dias, Y. V. Bludov, and N. M. R. Peres, Modeling the excitation of graphene plasmons in periodic grids of graphene ribbons: An analytical approach, Phys. Rev. B 94, 195421 (2016).
[34] Q. Xu, T. Ma, M. Danesh, B. N. Shivananju, S. Gan, J. Song, C.-W. Qiu, H.-M. Cheng, W. Ren, and Q. Bao, Effects of edge on graphene plasmons as revealed by infrared nanoimaging, Light Sci. Appl. 6, e16204 (2017).

[35] F. Hu, Y. Luan, Z. Fei, I. Z. Palubski, M. D. Goldflam, S. Dai, J.-S. Wu, K. W. Post, G. C. A. M. Janssen, M. M. Fogler, and D. N. Basov, Imaging the localized plasmon resonance modes in graphene nanoribbons, Nano Lett. 17, 5423 (2017).

[36] Z. Fei, M. D. Goldflam, J.-S. Wu, S. Dai, M. Wagner, A. S. McLeod, M. K. Liu, K. W. Post, S. Zhu, G. C. A. M. Janssen, M. M. Fogler, and D. N. Basov, Edge and surface plasmons in graphene nanoribbons, Nano Lett. 15, 8271 (2015).

[37] V. W. Brar, M. Seok Jang, M. Sherrott, S. Kim, J. J. Lopez, L. B. Kim, M. Choi, and H. Atwater, Hybrid surface-phononplasmon polariton modes in graphene/ monolayer h-BN heterostructures, Nano Lett. 14, 3876 (2014).

[38] L. Ju, B. Geng, J. Horng, C. Girit, M. Martin, Z. Hao, H. A. Bechtel, X. Liang, A. Zettl, Y. R. Shen, and F. Wang, Graphene plasmonics for tunable terahertz metamaterials, Nat. Nanotech. 6, 630 (2011).

[39] H. Yan, T. Low, W. Zhu, Y. Wu, M. Freitag, X. Li, F. Guinea, P. Avouris, and F. Xia, Damping pathways of mid-infrared plasmons in graphene nanostructures, Nat. Photon. 7, 394 (2013).

[40] V. W. Brar, M. S. Jang, M. Sherrott, J. J. Lopez, and H. A. Atwater, Highly confined tunable mid-infrared plasmonics in graphene nanoresonators, Nano Lett. 13, 2541 (2013).

[41] L. Brey and H. A. Fertig, Electronic states of graphene nanoribbons studied with the Dirac equation, Phys. Rev. B 73, 235411 (2006).

[42] A. H. Castro Neto, F. Guinea, N. M. R. Peres, K. S. Novoselov, and A. K. Geim, The electronic properties of graphene, Rev. Mod. Phys. 81, 109 (2009).

[43] B. Wunsch, T. Stauber, F. Sols, and F. Guinea, Dynamical polarization of graphene at finite doping, New J. Phys. 8, 318 (2006).

[44] K. T. Winther, Quantum theory of plasmons in nanostructures, Ph.D. thesis, Technical University of Denmark, 2015, http:// orbit.dtu.dk/en/publications/quantum-theory-of-plasmons-innanostructures(0f5c6bf0-7d18-4c13-b7ad-3895aaae4457). html.

[45] K. Andersen, K. W. Jacobsen, and K. S. Thygesen, Spatially resolved quantum plasmon modes in metallic nano-films from first-principles, Phys. Rev. B 86, 245129 (2012).

[46] T. Christensen, From Classical to Quantum Plasmonics in Three and Two Dimensions, Springer Theses (Springer International Publishing, Cham, 2017).

[47] K. A. Velizhanin, Geometric universality of plasmon modes in graphene nanoribbon arrays, Phys. Rev. B 91, 125429 (2015).

[48] E. H. Hwang and S. Das Sarma, Dielectric function, screening, and plasmons in two-dimensional graphene, Phys. Rev. B 75, 205418 (2007).

[49] S. Raza, S. I. Bozhevolnyi, M. Wubs, and N. A. Mortensen, Nonlocal optical response in metallic nanostructures, J. Phys.: Condens. Matter 27, 183204 (2015). 